\title{
Fat mass and obesity-associated protein promotes the tumorigenesis and development of liver cancer
}

\author{
ZIQI YE ${ }^{1 *}$, SHIBING WANG ${ }^{2 *}$, WANYUAN CHEN ${ }^{3}$, XIN ZHANG $^{3}$, JIE CHEN $^{4}$, \\ JINYING JIANG ${ }^{5}$, MINGSHAN WANG $^{6}$, LI ZHANG $^{7}$ and ZIXUE XUAN ${ }^{5}$
}

\begin{abstract}
${ }^{1}$ Department of Pharmacy, The First Affiliated Hospital, College of Medicine, Zhejiang University, Hangzhou, Zhejiang 310003; ${ }^{2}$ Key Laboratory of Tumor Molecular Diagnosis and Individualized Medicine of Zhejiang Province and Clinical Research Institute; ${ }^{3}$ Department of Pathology, Zhejiang Provincial People's Hospital, People's Hospital of Hangzhou Medical College, Hangzhou, Zhejiang 310014; ${ }^{4}$ Department of Pharmacy,

The Second Affiliated Hospital, College of Medicine, Zhejiang University, Hangzhou, Zhejiang 310009; Departments of ${ }^{5}$ Pharmacy and ${ }^{6}$ Infection Diseases, Zhejiang Provincial People's Hospital, People's Hospital of Hangzhou Medical College, Hangzhou, Zhejiang 310014; ${ }^{7}$ Department of Emergency Medicine, The Central Hospital of Lishui, Lishui, Zhejiang 323000, P.R. China
\end{abstract}

Received October 9, 2019; Accepted April 23, 2020

DOI: $10.3892 / 01.2020 .11673$

\begin{abstract}
Liver cancer is the fourth leading cause of cancer-associated mortality worldwide. Statistics indicate that the incidence of liver cancer has been increasing and that its prognosis remains poor. Fat mass and obesity-associated protein (FTO) is a demethylase that is involved in N6-methyladenosine (m6a) RNA modification; however, to the best of our knowledge, its role in tumorigenesis and development of liver cancer remains unknown. In the present study, cell proliferation, colony formation, apoptosis, Transwell and wound healing assays of small interfering (si)RNA-FTO HepG2 cells were performed, and the levels of m6A RNA methylation were assessed. Additionally, the prognostic value of FTO in liver cancer was analyzed using immunohistochemistry analysis. The results from the EpiQuik m6A RNA methylation quantitative assay revealed that knockdown of FTO increased the total m6A methylation level. Notably, FTO promoted the proliferation and migration of liver cancer cells. Additionally, FTO expression was upregulated in patients with liver cancer and was associated with a high Edmondson Grade, which served as an independent prognostic factor for liver cancer. Results from the Kaplan-Meier survival analysis
\end{abstract}

Correspondence to: Dr Zixue Xuan, Department of Pharmacy, Zhejiang Provincial People's Hospital, People's Hospital of Hangzhou Medical College, 158 Shangtang Road, Hangzhou, Zhejiang 310014, P.R. China

E-mail: xuanzixue0222@163.com

*Contributed equally

Key words: fat mass and obesity-associated protein, liver cancer, N6-methyladenosine modification, prognosis, proliferation revealed that low expression levels of FTO predicted a good prognosis. The 5-year overall survival of the low FTO expression group was $68 \%$ compared with $48 \%$ in the high FTO expression group $(\mathrm{P}=0.077)$. In conclusion, the present study suggested that FTO regulates the tumorigenesis and development of liver cancer.

\section{Introduction}

Liver cancer is the fifth and ninth most commonly diagnosed cancer globally in men and women, respectively (1). It was the fourth leading cause of cancer-associated mortality worldwide and was estimated to cause $\sim 800,000$ global deaths in 2015 (2). Liver cancer is frequently diagnosed at an advanced stage and is often characterized by poor prognosis (3). Consequently, only a small proportion of patients are eligible for potentially curative therapies (4). Various therapeutic strategies are currently used in the management of liver cancer, including surgery, liver transplantation, chemotherapy, radiotherapy and immunotherapy (5-12). However, liver cancer is frequently unresponsive to chemotherapy and radiotherapy, making its clinical outcomes poor.

The existence of chemically modified RNA species has been documented during the past decades (13). One of the most common chemical modifications on RNA molecules is the N6-methyladenosine (m6A) modification on mRNAs and long non-coding RNAs, which serve a crucial role in gene expression (14). This reversible RNA modification is catalyzed by the adenosine methyltransferases family of enzymes (15) and is reversed by demethylases (16). Members of the m6A methyltransferase family include methyltransferase like (METTL)3, METTL14, WT1 associated protein (WTAP), RNA binding motif protein 15 and vir like m6A methyltransferase associated (15). The m6A demethylase group of enzymes includes the fat mass and obesity-associated protein (FTO) and AlkB homolog 5 (16). Additionally, it has been demonstrated that 
m6A can be selectively recognized by proteins, including heterogeneous nuclear ribonucleoprotein $\mathrm{C}$, heterogeneous nuclear ribonucleoprotein A2/B1, YTH N6-methyladenosine RNA binding protein (YTHDF)2, YTHDF1 and eukaryotic translation initiation factor 3 subunit A (14). FTO has been demonstrated to modulate multiple RNA modifications, including m6A and N6,2-O-dimethyladenosine (17), and it has been recently reported that the activity of this enzyme is oncogenic in certain types of cancer $(18,19)$. Furthermore, FTO has been implicated in the induction of resistance to chemo-radiotherapy in cervical cancer by influencing the function of $\beta$-catenin via mRNA demethylation (20). Additionally, FTO upregulation has been associated with breast cancer progression via the PI3K/AKT signaling pathway (21). Overall, the aforementioned studies suggest that FTO may have various oncogenic roles in numerous types of cancer by modulating different cell signaling pathways.

The role of m6A methylation in the development of liver cancer has been explored in a few studies. For example, it has been reported that through a m6A-YTHDF2-dependent mechanism, METTL3 promotes tumorigenicity and metastasis of liver cancer in vitro and in vivo (22). Additionally, it has been proposed that WTAP serves an important role in the progression of liver cancer via m6A-HuR-dependent epigenetic silencing of the ETS proto-oncogene 1 (23). However, there is a lack of similar studies on the role of FTO in liver cancer. The aim of the present study was to explore the biological functions of FTO and its clinical relevance in liver cancer.

\section{Materials and methods}

Human samples, tissue microarray and cell lines. A total of 330 liver cancer tissues and 187 adjacent non-cancerous tissues ( $\geq 5 \mathrm{~cm}$ from the edge of the tumor tissue), were obtained from 330 patients at Zhejiang Provincial People's Hospital (Hangzhou, China). Written informed consent was obtained from all participants. The collected tissues were analyzed by FTO immunohistochemistry (IHC) and microarray analysis. The liver cancer tissue microarray was purchased from Shanghai BioChip Co., Ltd, and was performed according to the manufacturer's protocol. Ethical approval for the present study was obtained from the Ethics Committee of Zhejiang Provincial People's Hospital. The enrolled patients consisted of 268 males and 62 females, with a median age of 56 years (range, 25-91 years) at the time of surgery. Patient follow-up was performed for $\geq 5$ years and the survival time was calculated from the date of surgical intervention to death. The human liver cancer HepG2 cell line was purchased from the American Type Culture Collection. HepG2 cells were cultured in DMEM supplemented with 10\% FBS (both Gibco; Thermo Fisher Scientific, Inc.), $100 \mu \mathrm{g} / \mathrm{ml}$ penicillin and $0.1 \mathrm{mg} / \mathrm{ml}$ streptomycin at $37^{\circ} \mathrm{C}$ with $5 \% \mathrm{CO}_{2}$ in a humidified incubator.

Small interfering RNA (siRNA) transfection. HepG2 cells $\left(1 \times 10^{6}\right.$ cells/well) were seeded into 6-well plates and cultured with DMEM supplemented with $10 \% \mathrm{FBS}$ for $48 \mathrm{~h}$. Subsequently, cells were transfected with a mixture of siRNA $(100 \mathrm{nM})$ and Lipofectamine ${ }^{\circledR} 2000$ reagent (Thermo Fisher Scientific, Inc.), according to the manufacture's protocol. The sequences of
siRNAs used were as follows: siRNA-FTO-1, 5'-GGATGACTC TCATCTCGAA-3'; siRNA-FTO-2, 5'-GCTGAAATATCCTA AACTA-3'; siRNA-FTO-3, 5'-GTCACGAATTGCCCGA ACA-3'; and control siRNA, 5'-UUCUCCGAACGUGUCA CGU-3'. Cells were collected 24 or $48 \mathrm{~h}$ post-transfection for subsequent experimentation.

Western blot analysis. Western blot analysis was performed as previously described (24). The primary antibodies used in the present study were anti-FTO (1,1000; ab124892; Abcam) and anti- $\beta$-actin (1:200; ab115777; Abcam). Immunoreactive products were visualized using the ChemiDoc ${ }^{\mathrm{TM}}$ Touch Imaging system (Bio-Rad Laboratories, Inc.) and semi-quantified by densitometry using ImageJ software (version 1.50; National Institutes of Health).

Cell proliferation assay. The cell proliferation assays were performed using the Cell Counting Kit-8 (CCK-8; cat. no. CK04; Dojindo Molecular Technologies, Inc.) according to the manufacturer's protocol (23). Briefly, $200 \mu 1$ HepG2 cells or siRNA-FTO HepG2 cells $\left(2 \times 10^{4}\right)$ were seeded into 96-well cell culture-treated plates. Cells were then cultured for 48, 72 or $96 \mathrm{~h}$, after which $10 \mu \mathrm{l} \mathrm{CCK-8}$ was directly added into the culture medium in each well. Subsequently, cells were incubated at $37^{\circ} \mathrm{C}$ for $2 \mathrm{~h}$, and the absorbance was read at $450 \mathrm{~nm}$ using a microplate reader. Cell proliferation was measured in five wells for each experimental group.

Colony formation assay. A total of $1 \times 10^{2} \mathrm{HepG} 2$ or siRNA-FTO HepG2 cells were seeded into each well of a 6-well cell culture plate and cultured for 7 days. Subsequently, cells were washed with PBS and fixed with $4 \%$ paraformaldehyde, and then stained with $0.5 \%$ crystal violet. Cells were observed under an inverted light microscope (magnification, x10).

Apoptosis analysis. HepG2 or siRNA-FTO HepG2 cells $\left(1 \times 10^{5}\right.$ cells/well) were seeded in 24-well plates and cultured for $48 \mathrm{~h}$. After being washed with PBS twice and trypsinization, cells were resuspended in $500 \mu \mathrm{l}$ binding buffer supplemented with $5 \mu 1$ Annexin V-FITC (Nanjing KeyGen Biotech Co., Ltd.) and $5 \mu \mathrm{l}$ propidium iodide according to the manufacturer's protocol. Finally, the fluorescence intensity of the samples was determined by flow cytometry (EPICS XL-MCL; Beckman Coulter,Inc.). The number of apoptotic cells in each sample was analyzed using FCS Express version 3.0 (De Novo Software).

Transwell assay and wound healing assay. Matrigel (BD Biosciences) was thawed at $4^{\circ} \mathrm{C}$ overnight and diluted with DMEM. A total of $60 \mu \mathrm{l}$ diluted Matrigel was added in the upper chambers of a 24-well Transwell insert and incubated at $37^{\circ} \mathrm{C}$ for $30 \mathrm{~min}$. A total of $200 \mu 1 \mathrm{HepG} 2$ cells or siRNA-FTO HepG2 cells $\left(2 \times 10^{4}\right.$ cells/well $)$ in serum-free DMEM were seeded into the upper chamber. DMEM supplemented with $10 \%$ FBS $(600 \mu \mathrm{l})$ was added into the lower chamber and incubated for $24 \mathrm{~h}$ at $37^{\circ} \mathrm{C}$. Non-migrating cells on the top of the Transwell insert were removed using a cotton swab. The cell migration rate was analyzed using methanol and $0.3 \%$ crystal violet staining. Wound healing experiments were performed to investigate the effect of FTO on the migration ability of HepG2 cells. The initial HepG2 or siRNA-FTO HepG2 cell seeding 
density was $2 \times 10^{5}$ cells $/ \mathrm{cm}^{2}$. A scratch wound was made using a $10-\mu 1$ pipette tip. The cells were washed twice with PBS and then incubated with serum-free DMEM for 24 or $48 \mathrm{~h}$ at $37^{\circ} \mathrm{C}$. The cells were visualized under an inverted light microscope. The amount of wound healing was quantified using ImageJ software (version 1.50; National Institutes of Health).

Measurement of total m6A level. Total RNA from HepG2 cells was extracted and purified using the RNeasy Mini kit (Qiagen $\mathrm{GmbH}$ ), and the level of m6A RNA methylation was assessed using the EpiQuik M6A RNA Methylation Quantification kit (EpiGentek Group, Inc.) according to the manufacturer's protocol.

IHC analysis. The tissues were fixed in $10 \%$ buffered formalin for 6-12 $\mathrm{h}$ at room temperature, embedded in paraffin and cut into 4- $\mu \mathrm{m}$-thick sections. Sections were deparaffinized in xylene, rehydrated using a gradient of ethanol concentrations $(100,95,85$ and $75 \%)$ and boiled in $1 \mathrm{mM}$ TE buffer using a high-pressure cooker $\left(\geq 100^{\circ} \mathrm{C}\right)$ for $3 \mathrm{~min}$ for antigen retrieval. Subsequently, the sections were blocked with $3 \%$ hydrogen peroxide for $15 \mathrm{~min}$ at room temperature to inhibit endogenous peroxidase activity and incubated with $10 \%$ goat non-immune serum (Invitrogen; Thermo Fisher Scientific, Inc.) for $20 \mathrm{~min}$ at room temperature. The sections were then incubated with anti-FTO antibody (dilution, 1:100; cat. no. ab124892; Abcam) overnight at $4^{\circ} \mathrm{C}$ and with goat anti-rabbit IgG H\&L (HRP) (1:2,000; cat. no. ab205718; Abcam) at room temperature for $15 \mathrm{~min}$. This was followed by development using a DAB Substrate kit (Dako; Agilent Technologies, Inc.). IHC staining of FTO was scored by two independent pathologists using a light microscope (magnification, x200), based on the intensity and the proportion of positively stained cells. Specifically, staining intensity was evaluated with a grading system: 0 , negative; 1 , weak; 2 , moderate; and 3 , strong. The percentage of positive cells was scored as follows: 0 , no staining; $1,1-25 \%$ cells stained; $2,26-50 \%$ cells stained; $3,51-75 \%$ cells stained; and $4,>75 \%$ cells stained. The final score was obtained by multiplying the scores for intensity and percentage of positive cells.

FTO mRNA expression analysis. UALCAN (http://ualcan.path. uab.edu) is an online tool that uses The Cancer Genome Atlas RNA-sequencing and clinical data from 31 types of cancer. Additionally, it analyzes the relative expression levels of a query gene in various tumor sub-groups based on individual cancer stage, tumor grade, body weight or other clinicopathological features (25). In the present study, UALCAN was used to evaluate the mRNA expression levels of FTO in cancer and normal liver samples.

Statistical analysis. All statistical analyses were conducted using SPSS v13.0 (SPSS Inc.) and data are presented as the mean \pm SD of at least three individual experiments. An unpaired t-test was used to compare the differences between two groups. Comparisons among multiple groups were analyzed using one-way ANOVA followed by Tukey's post hoc test (all data met the assumption of homogeneity of variance). A $\chi^{2}$-test was used to assess the association between FTO expression and clinicopathological parameters. A multivariate survival analysis was performed to identify the factors associated with prognosis according to the Cox proportional hazards regression model. The association between FTO expression and overall survival (OS) was analyzed by the Kaplan-Meier method with a log-rank test. $\mathrm{P}<0.05$ (two-tailed) was considered to indicate a statistically significant difference.

\section{Results}

FTO expression is increased in liver cancer tissues. To investigate the role of FTO in liver cancer oncogenesis, the expression profile of FTO was characterized in liver cancer tissues and was compared with that in adjacent normal liver tissues by tissue microarrays. FTO scores were calculated with scores of 0-5 and 6-12 representing the low and high expression groups, respectively. The results of the IHC analysis revealed high FTO expression in 330 liver cancer tissues (126/330; 38.2\%), which was significantly higher than high FTO expression in 187 normal liver tissues $\left(46 / 187 ; 24.6 \% ; \chi^{2}=10.765 ; \mathrm{P}=0.001\right.$; data not shown). These results were in agreement with the outcome of an analysis using UALCAN that revealed that FTO expression was higher in liver cancer tissues compared with in normal liver tissues $(\mathrm{P}<0.001$; Fig. 1A). Overall, these observations suggested that FTO upregulation may be associated with the development of liver cancer.

FTO promotes in vitro proliferation and mobility of the human liver cancer cell line HepG2. FTO may be a modulator of m6A RNA demethylation and may promote liver cancer oncogenesis (17). siRNAs were used to knock down FTO expression in the human liver cancer HepG2 cell line. FTO expression downregulation was confirmed by western blotting (Fig. 1B and C). In addition, the m6A RNA level in the total RNA pool was quantified and it was observed that FTO knockdown was accompanied by a significant increase in the level of m6A RNA ( $\mathrm{P}=0.032$; Fig. 1D), suggesting an m6A demethylation function for FTO that may serve a role in liver cancer carcinogenesis. However, the exact mechanism needs to be further elucidated. Subsequently, a cell proliferation assay was performed using CCK-8. This analysis indicated that downregulation of FTO expression suppressed cell proliferation (Fig. 1E).

To further explore the role of this gene in liver cancer carcinogenesis, a colony formation assay was performed and it was observed that downregulation of FTO expression impaired the ability of HepG2 cells to form colonies(Fig. 2A and B). To establish the mechanism by which loss of FTO expression impaired these processes, an apoptosis assay was performed. Although transfection with siRNA-FTO appeared to decrease apoptosis, there was no significant difference in apoptosis between siRNA-NC and siRNA-FTO HepG2 cells, suggesting that FTO knockdown did not affect the apoptosis of HepG2 cells (Fig. 2C and D). Cell migration and wound healing assays suggested that FTO promoted the invasion and migration of liver cancer cells, as this was suppressed with FTO knockdown (Fig. 3). Overall, these findings indicated that FTO may promote the proliferation and migration of liver cancer cells in vitro.

Prognostic value of FTO in patients with liver cancer. The potential clinical prognostic value of FTO was investigated. 

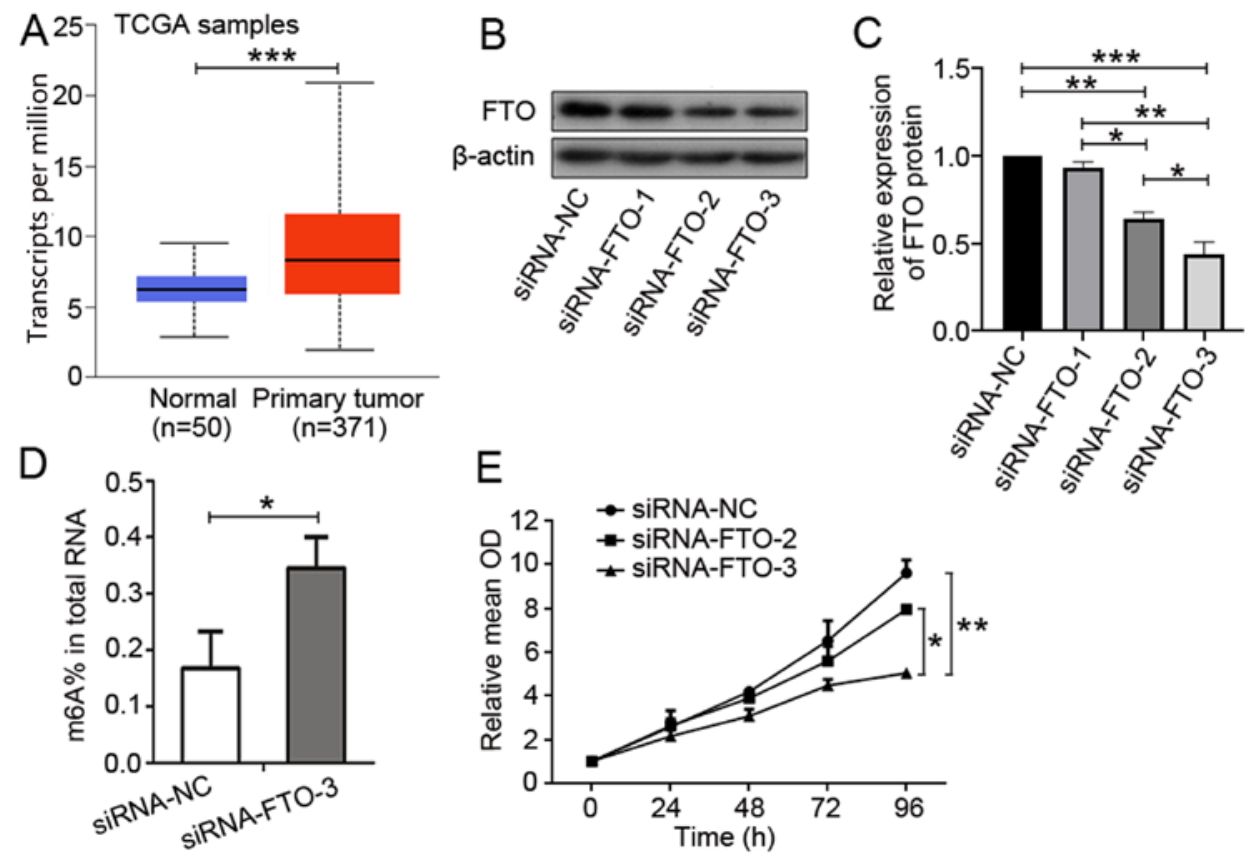

Figure 1. FTO promotes the proliferation of the HepG2 liver cancer cells in vitro. (A) TCGA database analysis by UALCAN revealed that FTO is highly expressed in liver cancer tissues compared with in normal liver tissues. (B) FTO protein downregulation was confirmed by western blotting. (C) Semi-quantified data of relative expression of FTO in siRNA-transfected cells. (D) Knockdown of FTO significantly upregulated the total RNA of m6A in HepG2 cells $(\mathrm{P}=0.032)$. (E) Knockdown of FTO inhibited the proliferation of HepG2 cells, as determined by CCK-8 assay. ${ }^{*} \mathrm{P}<0.05 ;{ }^{* *} \mathrm{P}<0.01 ;{ }^{* * *} \mathrm{P}<0.001 . \mathrm{TCGA}, \mathrm{The}$ Cancer Genome Atlas; NC, negative control; FTO, fat mass and obesity-associated protein; siRNA, small interfering RNA.

A
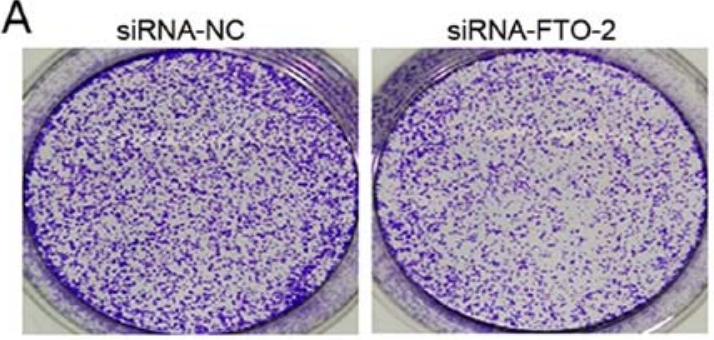

C
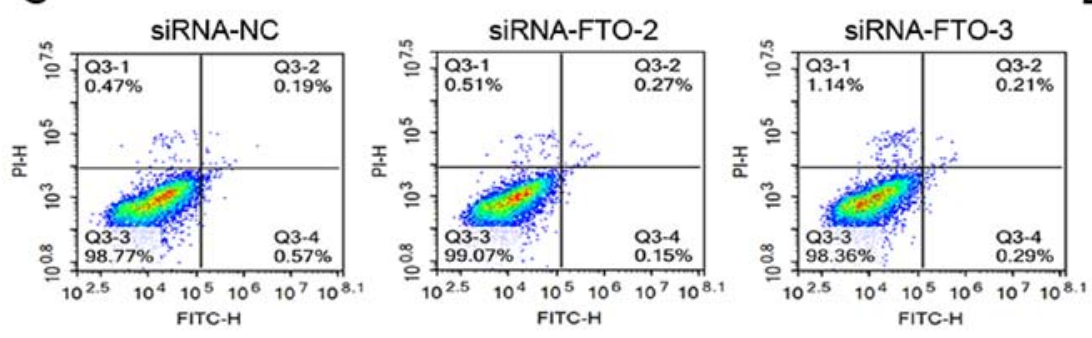
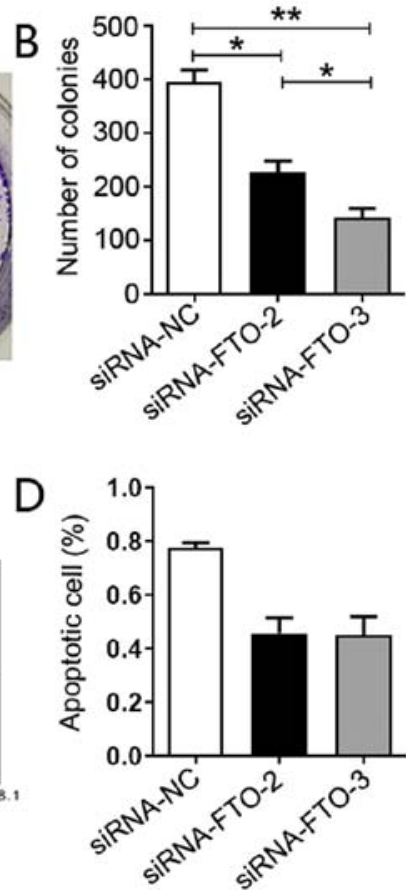

Figure 2. Knockdown of FTO inhibits the colony forming ability of HepG2 cells in vitro. (A) Downregulation of FTO expression suppressed the ability of HepG2 cells to form colonies. (B) Quantified data of the colony formation assay. (C) Knockdown of FTO expression had no effect on apoptosis of HepG2 cells. (D) Quantified data of the apoptosis assay. ${ }^{*} \mathrm{P}<0.05 ;{ }^{* *} \mathrm{P}<0.01$. NC, negative control; FTO, fat mass and obesity-associated protein; siRNA, small interfering RNA; PI, propidium iodide; FITC, fluorescein isothiocyanate.

The expression levels of FTO in patients with liver cancer at Zhejiang Provincial People's Hospital were analyzed using IHC and the association between FTO expression and 5 -year OS was explored. The results indicated a significantly higher FTO expression in liver cancer tissues compared with adjacent normal tissues (Fig. 4A; data not shown). Additionally, a significant positive association was identified between FTO expression and Edmonson Grade (4) $\left(\chi^{2}=10.523\right.$; $\mathrm{P}=0.001$; Table I), which was an established prognostic indicator for liver cancer in multivariate analysis (coefficient, 0.990; 


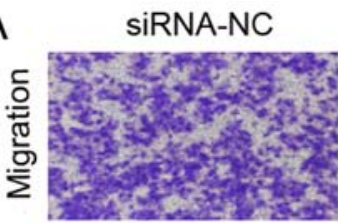

siRNA-FTO-2
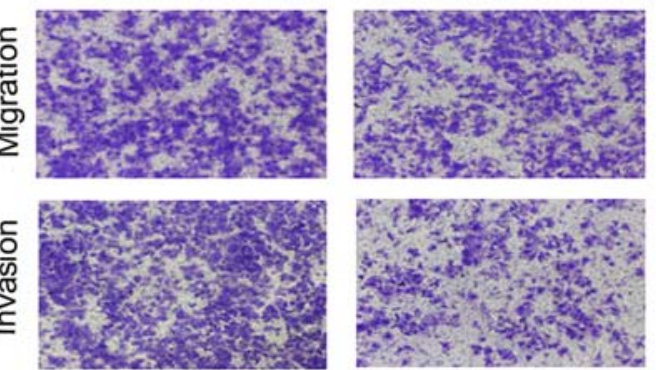

B
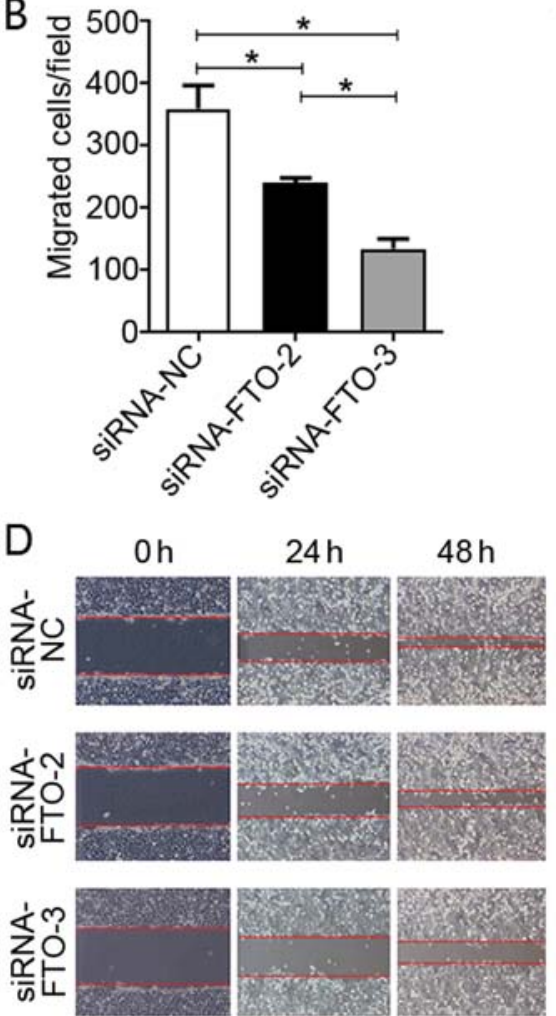
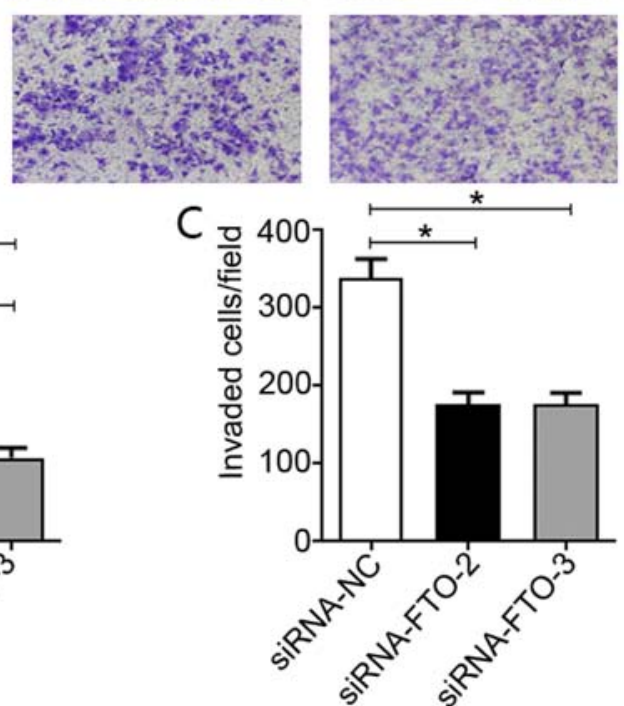

E $\square$ siRNA-NC siRNA-FTO-2 siRNA-FTO-3

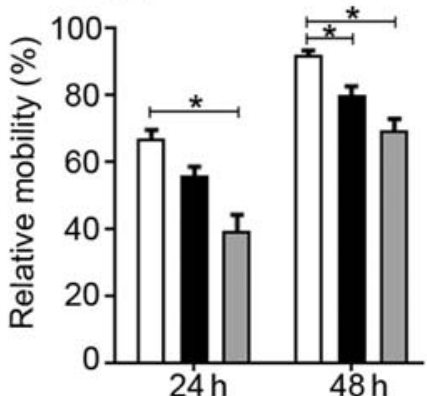

Figure 3. FTO promotes the migration of liver cancer cells. (A) Knockdown of FTO inhibited the invasion and migration of HepG2 cells (Transwell assay). Magnification, x10. Quantified data of the (B) migration and (C) invasion assays. (D) Downregulation of FTO expression inhibited the migration of HepG2 cells (wound healing assay). Magnification, x10. (E) Quantified data of the wound healing assays. * $\mathrm{P}<0.05$. NC, negative control; FTO, fat mass and obesity-associated protein; siRNA, small interfering RNA.

$\mathrm{P}=0.006$; Table II). The Kaplan-Meier analysis suggested that reduced FTO expression was indicative of a good prognosis. Although not statistically significant, a $68 \%$ 5-year OS was associated with low FTO expression compared with a $48 \%$ 5-year OS for high FTO expression ( $\mathrm{P}=0.077$; Fig. 4B).

\section{Discussion}

The present study reports a critical role of FTO in liver cancer tumorigenesis. The present analyses indicated that upregulation of FTO expression was frequently observed in liver cancer tissues. Knockdown of FTO significantly suppressed proliferation and migration of cultured liver cancer cells in vitro. Additionally, FTO knockdown led to a significant elevation of m6A methylation, suggesting that FTO-mediated m6A demethylation may contribute to liver cancer. However, the mechanism of this process remains unclear and merits further investigation. The IHC analysis revealed that FTO expression was markedly elevated in liver cancer tissues compared with adjacent normal tissues. Furthermore, a positive association between FTO expression and Edmonson Grade was observed, suggesting that FTO may be a potential prognostic indicator for liver cancer. The Kaplan-Meier analysis suggested that low FTO expression was indicative of good prognosis.

RNA methylation has recently emerged as an important modulator of tumorigenesis. m6A methylation is one of the most common modifications found in eukaryotic mRNA and is a critical event in RNA metabolism (26). It is becoming increasingly evident that the 'writers', 'erasers' and 'readers' of m6A serve critical roles in tumorigenesis (27). FTO has been identified as a m6A 'eraser' and is associated not only with increased body mass and obesity (28), but also with carcinogenesis (29). The association between FTO single nucleotide polymorphisms and tumorigenesis has been previously investigated in prostate, pancreatic, breast and colorectal cancer $(29,30)$. However, the m6A demethylase role of FTO in cancer development has only recently emerged. It has been previously demonstrated that FTO expression is enriched in 
Table I. FTO expression in liver cancer tissues.

\begin{tabular}{|c|c|c|c|c|c|}
\hline \multirow[b]{2}{*}{ Clinical parameters } & \multirow[b]{2}{*}{ Total no. } & \multicolumn{2}{|c|}{ FTO expression, $\mathrm{n}$} & \multirow[b]{2}{*}{$\chi^{2}$} & \multirow[b]{2}{*}{ P-value } \\
\hline & & Low & High & & \\
\hline Age, years & & & & 0.190 & 0.663 \\
\hline$<55$ & 128 & 81 & 47 & & \\
\hline$\geq 55$ & 202 & 123 & 79 & & \\
\hline Sex & & & & 0.456 & 0.500 \\
\hline Male & 268 & 168 & 100 & & \\
\hline Female & 62 & 36 & 26 & & \\
\hline Size, cm & & & & 1.328 & 0.249 \\
\hline$\leq 5$ & 192 & 123 & 69 & & \\
\hline$>5$ & 130 & 75 & 55 & & \\
\hline NA & 8 & & & & \\
\hline Tumour number & & & & 0.730 & 0.393 \\
\hline Single & 270 & 164 & 106 & & \\
\hline Multiple & 60 & 40 & 20 & & \\
\hline Edmondson grade & & & & 10.523 & $0.001^{\mathrm{a}}$ \\
\hline $\mathrm{I}+\mathrm{II}$ & 205 & 139 & 66 & & \\
\hline III & 119 & 59 & 60 & & \\
\hline NA & 6 & & & & \\
\hline Metastasis & & & & 0.525 & 0.469 \\
\hline M0 & 297 & 186 & 111 & & \\
\hline M1 & 27 & 15 & 12 & & \\
\hline NA & 6 & & & & \\
\hline Microvascular invasion & & & & 3.449 & 0.063 \\
\hline Absence & 124 & 83 & 41 & & \\
\hline Presence & 121 & 67 & 54 & & \\
\hline NA & 85 & & & & \\
\hline HBs antigen & & & & 1.135 & 0.287 \\
\hline Negtive & 63 & 35 & 28 & & \\
\hline Positive & 261 & 164 & 97 & & \\
\hline NA & 6 & & & & \\
\hline Cirrhosis & & & & 0.231 & 0.631 \\
\hline Negative & 110 & 66 & 44 & & \\
\hline Positive & 220 & 138 & 82 & & \\
\hline $\mathrm{AFP}, \mu \mathrm{g} / \mathrm{l}$ & & & & 2.056 & 0.152 \\
\hline$<50$ & 145 & 92 & 53 & & \\
\hline$\geq 50$ & 124 & 68 & 56 & & \\
\hline NA & 61 & & & & \\
\hline
\end{tabular}

FTO expression was divided into low and high expression groups based on immunohistochemistry scores of 0-5 and 6-12, respectively. ${ }^{\mathrm{a}} \mathrm{P}<0.05$. FTO, fat mass and obesity-associated protein; HBs, hepatitis B surface; AFP, $\alpha$-fetoprotein; NA, not available.

the tumor area and that it promotes proliferation, migration and lymph node metastasis in gastric cancer (19). In addition, Li et al (31) revealed that FTO expression is associated with an overall decrease in cancer survival. It has also been reported that FTO significantly enhances proliferation while inhibiting apoptosis in lung cancer cell lines (32).

Mechanistically, FTO has been demonstrated to suppress the m6A methylation of ubiquitin specific peptidase 7 while increasing mRNA stability via mRNA demethylation (33). These results indicate that FTO may promote oncogenesis and that suppressing its expression may offer a beneficial therapeutic strategy for various types of cancer. The results of the present study revealed that FTO may promote proliferation and migration of the liver cancer HepG2 cell line in vitro. However, the wound healing assay was inconclusive as it could not exclude the possibility that the suppressed 
Table II. Univariate and multivariate Cox regression analyses for the clinicopathological parameters in patients with liver cancer.

\begin{tabular}{|c|c|c|c|c|c|c|c|c|c|}
\hline \multirow[b]{2}{*}{ Parameters } & \multirow[b]{2}{*}{ No. } & \multicolumn{4}{|c|}{ Univariate analysis } & \multicolumn{4}{|c|}{ Multivariate analysis } \\
\hline & & Coefficient & HR & $95 \% \mathrm{CI}$ & P-value & Coefficient & HR & $95 \% \mathrm{CI}$ & P-value \\
\hline Age $(<55 / \geq 55$ years $)$ & $128 / 202$ & -0.427 & 0.653 & $0.412-1.034$ & 0.069 & -0.275 & 0.759 & $0.381-1.514$ & 0.435 \\
\hline Sex (male/female) & $268 / 62$ & 0.414 & 1.512 & $0.888-2.577$ & 0.128 & -0.194 & 0.823 & $0.384-1.766$ & 0.618 \\
\hline Tumor size $(\leq 50 />50 \mathrm{~mm})$ & $192 / 130$ & 0.714 & 2.042 & $1.283-3.248$ & $0.003^{\mathrm{a}}$ & 0.448 & 1.565 & $0.729-3.356$ & 0.250 \\
\hline Tumor number (single/multiple) & $270 / 60$ & 0.160 & 1.173 & $0.644-2.138$ & 0.601 & 0.860 & 2.364 & $0.982-5.692$ & 0.055 \\
\hline Edmondson grade (I+II/III) & $205 / 119$ & 1.018 & 2.769 & $1.733-4.423$ & $0.000^{\mathrm{a}}$ & 0.990 & 2.691 & $1.319-5.490$ & $0.006^{\mathrm{a}}$ \\
\hline Metastasis (M0/M1) & $297 / 27$ & 1.402 & 4.063 & $2.173-7.596$ & $0.000^{\mathrm{a}}$ & 1.424 & 4.153 & $1.481-9.625$ & $0.007^{\mathrm{a}}$ \\
\hline Microvascular invasion (-/+) & $124 / 121$ & 0.637 & 1.891 & $1.136-3.148$ & $0.041^{\mathrm{a}}$ & -0.170 & 0.844 & $0.382-1.862$ & 0.674 \\
\hline HBs antigen $(-/+)$ & $63 / 261$ & 0.125 & 1.133 & $0.633-2.030$ & 0.674 & -0.173 & 0.841 & $0.267-2.652$ & 0.768 \\
\hline Cirrhosis (-/+) & $110 / 220$ & 0.167 & 1.182 & $0.717-1.948$ & 0.513 & 0.781 & 2.183 & $0.881-5.406$ & 0.092 \\
\hline $\operatorname{AFP}(<50 / \geq 50 \mu \mathrm{g} / \mathrm{l})$ & $145 / 124$ & 0.837 & 2.310 & $1.315-4.058$ & $0.004^{\mathrm{a}}$ & 0.504 & 1.655 & $0.801-3.418$ & 0.174 \\
\hline FTO (-/+) & $185 / 124$ & 0.412 & 1.150 & $0.950-2.399$ & 0.081 & 0.451 & 1.570 & $0785-3.141$ & 0.202 \\
\hline
\end{tabular}

The total number of patients for some parameters was $<330$ due to incomplete pathological data. ${ }^{\mathrm{a}} \mathrm{P}<0.05$. FTO, fat mass and obesity-associated protein; HBs, hepatitis B surface; AFP, $\alpha$-fetoprotein; HR, hazard ratio.

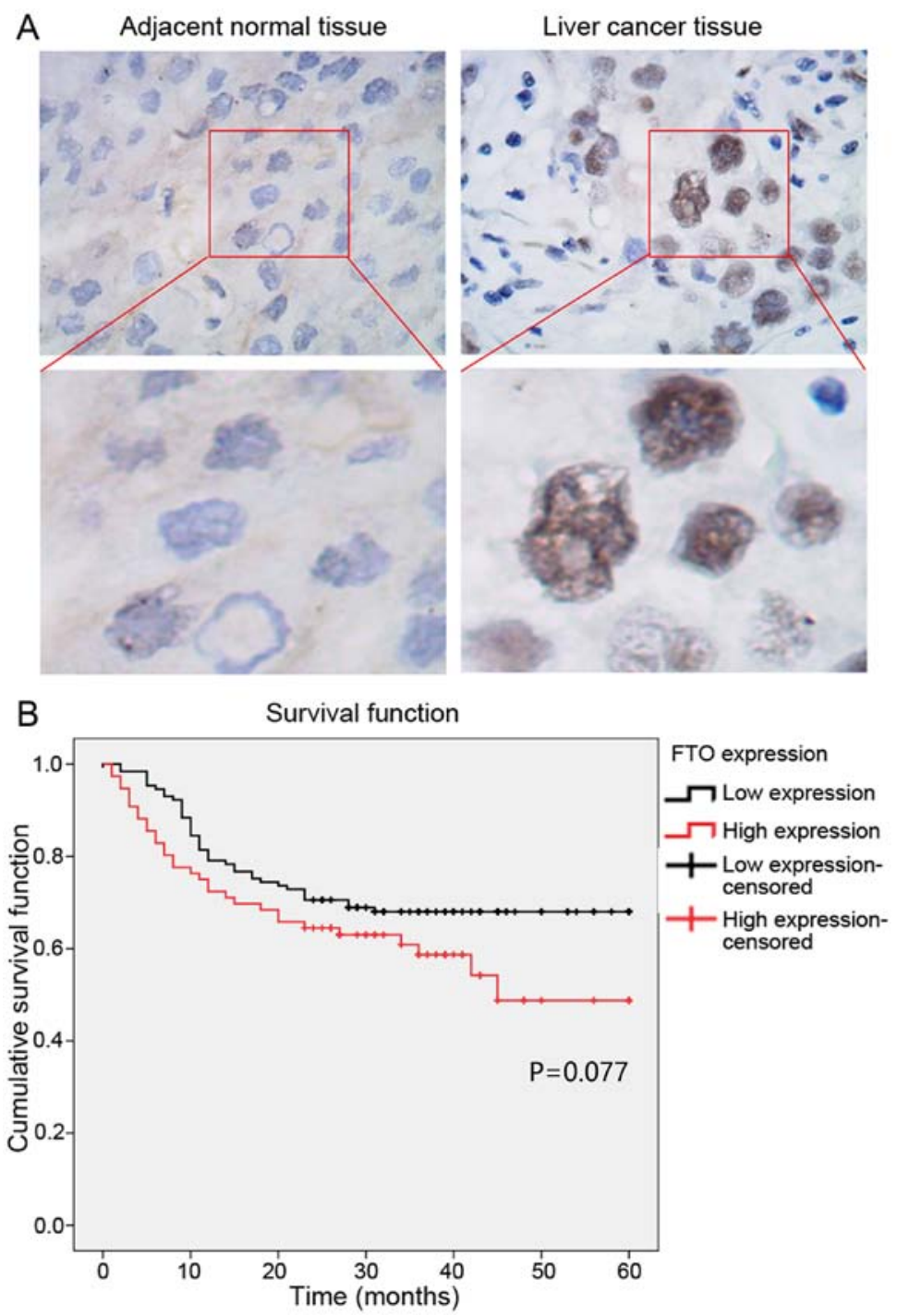

Figure 4. Prognostic value of FTO in liver cancer. (A) Representative IHC staining of FTO in specimens from patients with liver cancer. Magnification, x200. (B) Kaplan-Meier survival curves of patients with liver cancer with 'low FTO expression' (IHC score, 0-5) and 'high FTO expression' (IHC score, 6-12). FTO, fat mass and obesity-associated protein; IHC, immunohistochemistry. 
migration observed upon FTO knockdown was not caused by a reduction in cell proliferation. Results from the Transwell assay supported the aforementioned observation that reduced FTO expression may inhibit invasion and migration of HepG2 cells. Although FTO seemed to promote proliferation of HepG2 cells, it was also observed that this does not significantly affect apoptosis. Huang et al (33) demonstrated that FTO depletion decreases the expression levels of the mitotic checkpoint complex and $\mathrm{G} 2 / \mathrm{M}$ regulators in mouse $\mathrm{GC}-1$ cells. Wu et al (34) observed that FTO knockdown markedly decreases the expression levels of cyclin A2 and CDK2, both crucial cell cycle regulators, leading to delayed entry of methylene diphenyl diisocyanate-induced cells into G2 phase. However, whether FTO-knockdown affects the cell cycle of HepG 2 cells is unclear, and the mechanism of FTO promoting proliferation of HepG 2 cells is likely more complex than just through cell cycle arrest. Therefore, these mechanisms need to be further clarified. By suggesting a potential role of FTO in liver cancer, the results of the present study strengthened earlier reports of a role of FTO in oncogenesis. However, further research is required to elucidate the mechanism by which FTO-mediated modulation of m6A demethylation affects liver cancer oncogenesis.

The role served by m6A methylation in the development of liver cancer has been previously explored. It has been reported that the methyltransferase METTL3 promotes tumorigenicity and metastasis of liver cancer in vitro and in vivo by suppressing the expression of suppressor of cytokine signaling 2 via an m6A-YTHDF2-dependent mechanism (22). The m6A methyltransferase WTAP has also been reported to serve a crucial role in the development of liver cancer via the HuR-ETS1-p21/p27 axis (23). Although more evidence is required to support a role of FTO in liver cancer, it has been reported that FTO suppresses the proliferation and migration of intrahepatic cholangiocarcinoma cells by impairing the mRNA stability of the TEA domain transcription factor 2 oncogene (35). However, data from the present study revealed that FTO could promote the progression of liver cancer while downregulating m6A RNA methylation levels.

In conclusion, the present study revealed that FTO may contribute to liver cancer oncogenesis via the downregulation of m6A RNA methylation levels. Further investigations are required to better understand the association between the development of liver cancer and m6A methylation. The present study reported FTO as a potential prognostic indicator for liver cancer and a potential novel therapeutic target for the management of liver cancer.

\section{Acknowledgements}

Not applicable.

\section{Funding}

The present study was supported by the Project of Application on Public Welfare Technology in Zhejiang Province (grant no. LGF18H160022), the Joint Fund of Zhejiang Provincial Natural Science Foundation (grant no. LYY18H310002) and the Zhejiang Medical Technology Plan Project (grant no. 2017ZD003).

\section{Availability of data and materials}

The datasets used and/or analyzed during the current study are available from the corresponding author on reasonable request.

\section{Authors' contributions}

ZY, SW and ZX designed the study. ZY, SW, WC, XZ, JC, JJ, MW and LZ performed the data collection and collation. All the authors were involved in the analysis and interpretation of data. ZY wrote the paper, with the help of the co-authors. SW, WC and XZ reviewed and revised the manuscript. All authors agree to be accountable for all aspects of the research in ensuring that the accuracy or integrity of any part of the work are appropriately investigated and resolved. All authors read and approved the final manuscript.

\section{Ethics approval and consent to participate}

The present study was approved by the Ethics Committee of Zhejiang Provincial People's Hospital (Hangzhou, China). Written informed consent was obtained from all participants.

\section{Patient consent for publication}

Not applicable.

\section{Competing interests}

The authors declare that they have no competing interests.

\section{References}

1. Ferlay J, Soerjomataram I, Dikshit R, Eser S, Mathers C, Rebelo M, Parkin DM, Forman D and Bray F: Cancer incidence and mortality worldwide: Sources, methods and major patterns in GLOBOCAN 2012. Int J Cancer 136: E359-E386, 2015.

2. Akinyemiju T, Abera S, Ahmed M, Alam N, Alemayohu MA, Allen C, Al-Raddadi R, Alvis-Guzman N, Amoako Y, Artaman A, et al; Global Burden of Disease Liver Cancer Collaboration: The burden of primary liver cancer and underlying etiologies from 1990 to 2015 at the global, regional, and national level: Results from the global burden of Disease Study 2015. JAMA Oncol 3: 1683-1691, 2017.

3. Njei B, Rotman Y, Ditah I and Lim JK: Emerging trends in hepatocellular carcinoma incidence and mortality. Hepatology 61: 191-199, 2015.

4. Wang $\mathrm{H}, \mathrm{Lu} \mathrm{Z}$ and Zhao X: Tumorigenesis, diagnosis, and therapeutic potential of exosomes in liver cancer. J Hematol Oncol 12: 133, 2019.

5. Chok KS, Ng KK, Poon RT, Lo CM and Fan ST: Impact of postoperative complications on long-term outcome of curative resection for hepatocellular carcinoma. Br J Surg 96: 81-87, 2009.

6. Cha CH, Ruo L, Fong Y, Jarnagin WR, Shia J, Blumgart LH and DeMatteo RP: Resection of hepatocellular carcinoma in patients otherwise eligible for transplantation. Ann Surg 238: 315-321, discussion 321-323, 2003.

7. Riaz A, Miller FH, Kulik LM, Nikolaidis P, Yaghmai V, Lewandowski RJ, Mulcahy MF, Ryu RK, Sato KT, Gupta R, et al: Imaging response in the primary index lesion and clinical outcomes following transarterial locoregional therapy for hepatocellular carcinoma. JAMA 303: 1062-1069, 2010.

8. Riaz A, Ryu RK, Kulik LM, Mulcahy MF, Lewandowski RJ, Minocha J, Ibrahim SM, Sato KT, Baker T, Miller FH, et al: Alpha-fetoprotein response after locoregional therapy for hepatocellular carcinoma: Oncologic marker of radiologic response, progression, and survival. J Clin Oncol 27: 5734-5742, 2009.

9. Hawkins MA and Dawson LA: Radiation therapy for hepatocellular carcinoma: From palliation to cure. Cancer 106: 1653-1663, 2006. 
10. Palmer DH: Sorafenib in advanced hepatocellular carcinoma. N Engl J Med 359: 2498, author reply 2498-2499, 2008.

11. El-Khoueiry AB, Sangro B, Yau T, Crocenzi TS, Kudo M, Hsu C Kim TY, Choo SP, Trojan J, Welling TH III, et al: Nivolumab in patients with advanced hepatocellular carcinoma (CheckMate 040): An open-label, non-comparative, phase 1/2 dose escalation and expansion trial. Lancet 389: 2492-2502, 2017.

12. Voutsadakis IA: PD-1 inhibitors monotherapy in hepatocellular carcinoma: Meta-analysis and systematic review. Hepatobiliary Pancreat Dis Int 18: 505-510, 2019.

13. Machnicka MA, Milanowska K, Osman Oglou O, Purta E, Kurkowska M, Olchowik A, Januszewski W, Kalinowski S, Dunin-Horkawicz S, Rother KM, et al: MODOMICS: A database of RNA modification pathways - 2013 update. Nucleic Acids Res 41: D262-D267, 2013.

14. Dominissini D, Moshitch-Moshkovitz S, Schwartz S, Salmon-Divon M, Ungar L, Osenberg S, Cesarkas K, Jacob-Hirsch J, Amariglio N, Kupiec M, et al: Topology of the human and mouse m6A RNA methylomes revealed by m6A-seq. Nature 485: 201-206, 2012.

15. Liu J, Yue Y, Han D, Wang X, Fu Y, Zhang L, Jia G, Yu M, Lu Z, Deng X, et al: A METTL3-METTL14 complex mediates mammalian nuclear RNA N6-adenosine methylation. Nat Chem Biol 10: 93-95, 2014

16. Jia G, Fu Y, Zhao X, Dai Q, Zheng G, Yang Y, Yi C, Lindahl T, Pan T, Yang YG, et al: N6-methyladenosine in nuclear RNA is a major substrate of the obesity-associated FTO. Nat Chem Biol 7: 885-887, 2011

17. Bartosovic M, Molares HC, Gregorova P, Hrossova D, Kudla G and Vanacova S: N6-methyladenosine demethylase FTO targets pre-mRNAs and regulates alternative splicing and 3'-end processing. Nucleic Acids Res 45: 11356-11370, 2017.

18. Li Z, Weng H, Su R, Weng X, Zuo Z, Li C, Huang H, Nachtergaele S, Dong L, Hu C, et al: FTO Plays an Oncogenic Role in Acute Myeloid Leukemia as a N6-Methyladenosine RNA Demethylase. Cancer Cell 31: 127-141, 2017.

19. Xu D, Shao W, Jiang Y, Wang X, Liu Y and Liu X: FTO expression is associated with the occurrence of gastric cancer and prognosis. Oncol Rep 38: 2285-2292, 2017

20. Zhou S, Bai ZL, Xia D, Zhao ZJ, Zhao R, Wang YY and Zhe H: FTO regulates the chemo-radiotherapy resistance of cervical squamous cell carcinoma (CSCC) by targeting $\beta$-catenin through mRNA demethylation. Mol Carcinog 57: 590-597, 2018.

21. Liu Y, Wang R, Zhang L, Li J, Lou K and Shi B: The lipid metabolism gene FTO influences breast cancer cell energy metabolism via the PI3K/AKT signaling pathway. Oncol Lett 13 4685-4690, 2017.

22. Chen M, Wei L, Law CT, Tsang FH, Shen J, Cheng CL, Tsang LH, Ho DW, Chiu DK, Lee JM, et al: RNA N6-methyladenosine methyltransferase-like 3 promotes liver cancer progression through YTHDF2-dependent posttranscriptional silencing of SOCS2. Hepatology 67: 2254-2270, 2018.

23. Chen Y, Peng C, Chen J, Chen D, Yang B, He B, Hu W, Zhang Y, Liu H, Dai L, et al: WTAP facilitates progression of hepatocellular carcinoma via m6A-HuR-dependent epigenetic silencing of ETS1. Mol Cancer 18: 127, 2019.
24. Wang W, Liu L, Zhou Y, Ye Q, Yang X, Jiang J, Ye Z, Gao F, Tan X, Zhang G, et al: Hydroxychloroquine enhances the antitumor effects of $\mathrm{BC} 001$ in gastric cancer. Int J Oncol 55: 405-414, 2019.

25. Chandrashekar DS, Bashel B, Balasubramanya SA, Creighton CJ, Ponce-Rodriguez I, Chakravarthi BV and Varambally S: UALCAN: A portal for facilitating tumor subgroup gene expression and survival analyses. Neoplasia 19: 649-658, 2017.

26. Vu LP, Cheng Y and Kharas MG: The Biology of m6A RNA Methylation in Normal and Malignant Hematopoiesis. Cancer Discov 9: 25-33, 2019

27. Pan Y, Ma P, Liu Y, Li W and Shu Y: Multiple functions of m6A RNA methylation in cancer. J Hematol Oncol 11: 48, 2018.

28. Loos RJ and Bouchard C: FTO: The first gene contributing to common forms of human obesity. Obes Rev 9: 246-250, 2008.

29. Nock NL, Plummer SJ, Thompson CL, Casey G and Li L: FTO polymorphisms are associated with adult body mass index (BMI) and colorectal adenomas in African-Americans. Carcinogenesis 32: 748-756, 2011.

30. Jafari Nedooshan J, Kargar S, Neamatzadeh H, Haghighi F, Dehghani Mohammad Abadi R and Seddighi N: Lack of association of the fat mass and obesity associated (FTO) gene rs9939609 polymorphism with breast cancer risk: A systematic review and meta-analysis based on case - control studies. Asian Pac J Cancer Prev 18: 1031-1037, 2017.

31. Li Y, Zheng D, Wang F, Xu Y, Yu H and Zhang H: Expression of demethylase genes, FTO and ALKBH1, is associated with prognosis of gastric cancer. Dig Dis Sci 64: 1503-1513, 2019.

32. Li J, Han Y, Zhang H, Qian Z, Jia W, Gao Y, Zheng H and Li B The m6A demethylase FTO promotes the growth of lung cancer cells by regulating the m6A level of USP7 mRNA. Biochem Biophys Res Commun 512: 479-485, 2019.

33. Huang T, Gao Q, Feng T, Zheng Y, Guo J and Zeng W: FTO knockout causes chromosome instability and $\mathrm{G} 2 / \mathrm{M}$ arrest in mouse GC-1 cells. Front Genet 9: 732, 2019.

34. Wu R, Liu Y, Yao Y, Zhao Y, Bi Z, Jiang Q, Liu Q, Cai M, Wang F, Wang Y, et al: FTO regulates adipogenesis by controlling cell cycle progression via m6A-YTHDF2 dependent mechanism. Biochim Biophys Acta Mol Cell Biol Lipids 1863: 1323-1330, 2018.

35. Rong ZX, Li Z, He JJ, Liu LY, Ren XX, Gao J, Mu Y, Guan YD, Duan YM, Zhang XP, et al: Downregulation of fat mass and obesity associated (FTO) promotes the progression of intrahepatic cholangiocarcinoma. Front Oncol 9: 369, 2019.

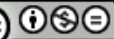

This work is licensed under a Creative Commons Attribution-NonCommercial-NoDerivatives 4.0 International (CC BY-NC-ND 4.0) License. 